\title{
RETRIEVAL OF LOCAL INTERPLANETARY DUST EMISSIVITY BY ASTRO-F
}

\author{
S. S. HONG ${ }^{1,5}$, S. M. KWON ${ }^{2}$, J. PYO ${ }^{1}$, \\ M. Ueno ${ }^{3}$, M. Ishiguro ${ }^{4}$, F. Usui $^{5}$, AND J. L. WeINBerg ${ }^{6}$ \\ ${ }^{1}$ Astronomy Program, SEES, Seoul National University, Seoul, Korea; \\ E-mail: sshong@astroism.snu.ac.kr,jhpyo@astro.snu.ac.kr \\ ${ }^{2}$ Department of Science Education, Kangwon National University, Chunchon, Korea; \\ E-mail: smkwon@cc.kangwon.ac.kr \\ ${ }^{3}$ Graduate School of Astronomy and Earth Sciences, University of Tokyo, Tokyo, Japan; \\ E-mail: ueno@provence.c.u-tokyo.ac.jp \\ ${ }^{4}$ Institute for Astronomy, University of Hawaii, Honolulu, Hawaii, USA; \\ E-mail: ishiguro@ifa.hawaii.edu \\ ${ }^{5}$ Department of IR Astrophysics, ISAS/JAXA, Sagamihara, Kanagawa, Japan; \\ E-mail: usui@ir.isas.jaxa.jp,sshong@ir.isas.jaxa.jp \\ ${ }^{6}$ Space Astronomy Lab, Snellville, GA 30039, USA; \\ E-mail: wein2@mindspring.com \\ (Received December 1, 2004; Accepted December 21,2004)
}

\begin{abstract}
This is a proposal to probe local part of the interplanetary dust (IPD) cloud complex and retrieve mean volume emissivity of the local IPDs at mid-infrared wavelengths. This will be done by monitoring, with Infrared Camera (IRC) aboard the ASTRO-F, the annual modulation of the zodiacal emission. In pointing mode of the ASTRO-F mission the spacecraft can make attitude maneuvering over approximately $\pm 1^{\circ}$ range centered at solar elongation $90^{\circ}$ in the ecliptic plane. The attitude maneuvering combined with high sensitivity of the IRC will provide us with a unique opportunity observationally to take derivatives of the zodiacal emission brightness with respect to the solar elongation. From the resulting differential of the brightness over the $\pm 1^{\circ}$ range, one can directly determine the mean volume emissivity of the local IPDs with a sufficient accuracy to de-modulate the annual emissivity variations due to the Earth's elliptical motion and the dis-alignment of the maximum IPD density plane with respect to the ecliptic. The non-zero eccentricity $\left(e_{\oplus}=0.0167\right)$ of the Earth's orbit combined with the sensitive temperature dependence of the Planck function would bring modulations of amplitude at least $3.34 \%$ to the zodiacal emission brightness at mid-infrared wavelengths, with which one may determine the IPD temperature $T(r)$ and mean number density $n(r)$ as functions of heliocentric distance $r$. This will in turn fix the power-law exponent $\delta$ in the relation $T(r)=T_{0}\left(r / r_{\mathrm{o}}\right)^{-\delta}$ for the dust temperature and $\nu$ in $n(r)=n_{\mathrm{o}}\left(r / r_{\mathrm{o}}\right)^{-\nu}$ for the density. We discuss how one may de-couple the notorious degeneracy of cross-section, density, reference temperature $T_{\mathrm{o}}$ and exponent $\delta$.
\end{abstract}

Key words : zodiacal emission ; interplanetary dusts ; IR emissivity ; density-temperature degeneracy

\section{INTRODUCTION}

Ever since Hiro Tanabe (1963), Jerry L. Weinberg (1963) and Rène Dumont (1965) observed light of the night sky painstakingly with photo-multipliers then the most modern light detector, we have been witnessing increasing details in the interplanetary dust (IPD) distribution. The birth of Infrared Astronomical Satellite (IRAS) in the early 1980s dramatically changed then the smooth featureless picture of the zodiacal dust cloud by revealing, in the all-sky distribution of the zodiacal emission (ZE), numerous bands of asteroidal debris near the ecliptic plane (Low et al. 1984; Dermott et al. 1984, 1985; Sykes 1988) and narrow trails of cometary dust (Davies et al. 1984; Sykes \& Walker

Corresponding Author: S. S. Hong
1992; Ishiguro et al. 2002a), and also by hinting us an existence of clumpy circumsolar dust ring embedded in the Earth's orbit (Reach 1991; Dermott et al. 1994). All these discoveries benefit from space-platform of the IRAS, which is free from the scattering and airglow emitting Earth's atmosphere. Until the mid 1980s the best available sky map of the zodiacal light (ZL) had $10 \sim 15 \%$ accuracy in the brightness measurement and $5^{\circ}$ resolution in the spatial brightness distribution (Lavasseur-Regourd \& Dumont 1980). By improving the accuracy and resolution achieved in the visible ZL to a few percents and a few tenths of a degree, respectively, in the infrared ZE, the IRAS has re-named the zodiacal dust cloud as the IPD cloud complex. In the early 1990s the Diffuse InfraRed Background Experiment (DIRBE) instrument on the COsmic Background Explorer (COBE) Satellite mapped the diffuse 
background over almost the entire sky. Although the DIRBE/COBE employed a $0.7^{\circ}$-wide beam, it confirmed the existence of the circumsolar ring of dusts trapped in the Earth's mean motion resonance (MMR), and isolated the leading and trailing blobs in the MMR feature (Reach et al. 1995; Kelsall et al. 1998). On entering the 21st century, the ground-based observation with a wide-field, small-aperture, cooled CCD camera (Ishiguro et al. 2003; Kwon et al. 2004) and deep photometry with a large-aperture Schmidt telescope (Ishiguro et al. 2003) began to show, respectively, the asteroidal debris bands (Ishiguro et.al. 1999) and cometary dust trails (Ishiguro et al. 2002a) in the visible. Most recently, Kwon, Hong and Weinberg (2004) found visible counter-parts of the MMR features in their newly reduced map of the ZL brightness. They also noticed from the map hints of additional debris bands at high ecliptic latitudes.

In spite of all these findings we still do not know the mean volume scattering cross-section of the local IPDs at visible wavelength quantitatively and the mean volume emission cross-section at infrared wavelengths either. The currently available values for these optical properties are not from direct measurements. They are either simple estimates (Hong 1985) or dependent on the model of three dimensional density distribution (Kwon \& Hong 1998). Furthermore, we haven't had a means to measure the mean temperature of even the local IPDs that are residing very close to the Earth's orbit. Uncertainties in the heliocentric distance dependence of the mean dust temperature are even worse than in the mean temperature itself.

As regard to probing the IPD cloud complex, we are now entering an exciting era. The InfraRed Camera (IRC) aboard the ASTRO-F will soon determine the ZE brightness at mid-infrared wavelengths with relative accuracy a few hundredths of a percent (cf. ASTRO-F Observing Manual Ver.1.5, March 31, 2002;

www.ir.isas.jaxa.jp/ASTRO-F/Observation/). On the other hand, eccentricity of the Earth's orbit is not zero but amounts to 0.0167 , which will modulate in a year period the heliocentric distance of the ASTRO-F by in total $0.0334 \mathrm{AU}$. Therefore, $0.0334 \mathrm{AU}$-wide region of band centered at $1 \mathrm{AU}$ from the Sun can be fully probed by the ASTRO-F during the 1.5 year period of its operation. The eccentricity modulation will bring about, over one year period, at least $3.34 \%$ change to the brightness of the ZE at fixed solar elongation. The high IRC sensitivity requires us to be conscientious about the eccentricity of the Earth's orbit; no matter how tiny it may be, it will render us some detectable changes in the observed brightness of ZE. This study aims at projecting how the ASTRO-F mission can probe the IPD cloud complex. In the present study a full use is going to be made of the Earth's elliptical path. Although the mission's coverage of the solar elongation angle is limited to a narrow span (cf. ASTRO-F Observing Manual Ver.1.5), we will fully utilize the spacecraft's attitude maneuvering capability in the probing.
In the next section $(\S 2)$ a detailed strategy will be outlined for the retrieval of physical and optical properties of the local IPDs and of their spatial distribution. For an easy understanding we will first consider a case of a hypothetical Earth's circular orbit, and then discuss useful complications brought by the Earth's elliptical motion. In the same section we will discuss how severe the MMR interference could be and suggest possible means to cope with it. In $\S 3$ we will examine whether the detection sensitivity of the IRC is sufficiently high to make our efforts rewarding. This will suggest that the required sensitivity can be safely achieved in the pointing mode of the ASTRO-F mission. In $\S 4$ we will perform a series of feasibility tests for our proposal. The probing of the IPD cloud will be done by de-modulating the annual variations of the observed ZE brightness in terms of the Earth's elliptical motion and an inclination of the maximum IPD density plane with respect to the ecliptic. For the tests we will introduce a model of the IPD cloud and simulate the resulting distribution of the $\mathrm{ZE}$ brightness at various time epochs. The final section concludes the present study with some discussions.

\section{STRATEGY FOR RETRIEVING LOCAL IPD PROPERTIES}

\section{(a) A Hypothetical Circular Orbit for Earth}

Let us assume that Earth orbits the Sun in a $1 \mathrm{AU}-$ radius circle. This simplification will help us understand how the local emissivity can be obtained by $\Lambda$ differentiation method. When the hypothetical Earth arrives at point A, whose ecliptic longitude is $\Lambda_{\mathrm{k}}-\delta \Lambda$, ASTRO-F is supposed to place her telescope axis in the ecliptic plane and to observe the sky brightness, $I_{\mathrm{A}}^{\mathrm{L}}\left(\Lambda_{\mathrm{k}}\right)$, in the direction that leads the Earth's motion and is off the usual $90^{\circ}$ solar elongation by a small angle $\delta \Lambda$. In other words the observing sight line has the solar elongation angle of $\epsilon=\pi / 2-\delta \Lambda$. As illustrated in Figure 1, some time later the Earth arrives at point $\mathrm{B}$, where ecliptic longitude is $\Lambda_{\mathrm{k}}+\delta \Lambda$, and the telescope observes the sky brightness, $I_{\mathrm{B}}^{\mathrm{L}}\left(\Lambda_{\mathrm{k}}\right)$, in the same leading direction but now making the elongation angle $\epsilon=\pi / 2+\delta \Lambda$. As done in the leading direction, we make the same set of measurements at $A$ and $B$ but in the direction trailing the Earth's motion. If the same notation is kept as in the leading direction, we may denote the resulting brightness measurements at $\mathrm{A}$ and $\mathrm{B}$ by $I_{\mathrm{A}}^{\mathrm{T}}\left(\Lambda_{\mathrm{k}}\right)$ and $I_{\mathrm{B}}^{\mathrm{T}}\left(\Lambda_{\mathrm{k}}\right)$, respectively. Measuring the sky brightness in two opposite directions at two nearby locations, whose mid-point has ecliptic longitude, say, $\Lambda_{\mathrm{k}}$, will generate a set of four brightness values $\left(I_{\mathrm{A}}^{\mathrm{L}}, I_{\mathrm{A}}^{\mathrm{T}}, I_{\mathrm{B}}^{\mathrm{L}}, I_{\mathrm{B}}^{\mathrm{T}}\right)_{\Lambda=\Lambda_{\mathrm{k}}}$. Over one year period we will obtain the same set of four measurements at a number of carefully selected locations along the orbit, i.e., $\mathrm{k}=1,2,3, \ldots, \mathrm{k}_{\max }$.

The dust particles in a line of observing sight consist of the following three components: The first is those 


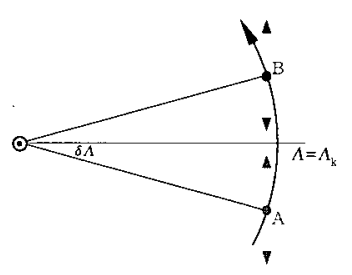

Fig. 1.- Geometry involved in the $\Lambda$-differentiation of $\mathrm{ZE}$ brightness. The thick arrow represents the direction of Earth's orbital motion. In each observing session four measurements are obtained, in the leading and trailing directions at each of the two locations $\Lambda_{k} \pm \delta \Lambda$. The difference between two brightness measurements made in the same leading or trailing directions is a simple product of the chord length $\overline{A B}$ and the mean volume emissivity of the local IPDs along the chord.

IPDs that are distributed rather smoothly in the solar system and act as the source of ZE, the second is the IPDs that are loosely trapped in the Earth's mean motion resonance (MR) and cause asymmetry in the ZE brightness at $90^{\circ}$ elongation between the leading and trailing directions, and the third is the interstellar and intergalactic dusts that comprise diffuse Galactic and extra-galactic background emissions (BG). Each of the four brightness measurements taken in an observing session $\Lambda_{\mathrm{k}}$ can be decomposed into the three components as:

$$
I_{\mathrm{A}}^{\mathrm{L}}\left(\Lambda_{\mathrm{k}}\right)=Z E_{\mathrm{A}}^{\mathrm{L}}(\pi / 2-\delta \Lambda)+M R_{\mathrm{A}}^{\mathrm{L}}(\pi / 2-\delta \Lambda)+B G_{\mathrm{A}}^{\mathrm{L}},
$$

$I_{\mathrm{A}}^{\mathrm{T}}\left(\Lambda_{\mathrm{k}}\right)=Z E_{\mathrm{A}}^{\mathrm{T}}(\pi / 2+\delta \Lambda)+M R_{\mathrm{A}}^{\mathrm{T}}(\pi / 2+\delta \Lambda)+B G_{\mathrm{A}}^{\mathrm{T}}$,

$$
I_{\mathrm{B}}^{\mathrm{L}}\left(\Lambda_{\mathrm{k}}\right)=Z E_{\mathrm{B}}^{\mathrm{L}}(\pi / 2+\delta \Lambda)+M R_{\mathrm{B}}^{\mathrm{L}}(\pi / 2+\delta \Lambda)+B G_{\mathrm{B}}^{\mathrm{L}},
$$

$I_{\mathrm{B}}^{\mathrm{T}}\left(\Lambda_{\mathrm{k}}\right)=Z E_{\mathrm{B}}^{\mathrm{T}}(\pi / 2-\delta \Lambda)+M R_{\mathrm{B}}^{\mathrm{T}}(\pi / 2-\delta \Lambda)+B G_{\mathrm{B}}^{\mathrm{T}}$,

where the solar elongation angles are specified for the smooth portion of ZE and MMR component, and for the observed intensities $\Lambda_{\mathbf{k}}$ is given to identify the observing session.

One may notice that $B G_{\mathrm{A}}^{\mathrm{L}}=B G_{\mathrm{B}}^{\mathrm{L}}$ and $B G_{\mathrm{A}}^{\mathrm{T}}=B G_{\mathrm{B}}^{\mathrm{T}}$ hold true for the background brightness. As long as the viewing direction is kept the same, it doesn't matter where the observation is made. For the $\mathrm{ZE}$ component, however, observing site does matter, even if the sight line points the same sky position. The ZE measured at $\mathrm{A}$ in the leading direction ought to be a little stronger than that at $B$ in the same direction, since the IPDs along chord $\overline{A B}$ render an additional contribution to $Z E_{\mathrm{A}}^{\mathrm{L}}$ over $Z E_{\mathrm{B}}^{\mathrm{L}}$. Aiming at the differential brightness, we subtract eq. (3) from eq. (1) to have

$$
\begin{gathered}
I_{\mathrm{A}}^{\mathrm{L}}-I_{\mathrm{B}}^{\mathrm{L}}=Z E_{\mathrm{A}}^{\mathrm{L}}(\pi / 2-\delta \Lambda)-Z E_{\mathrm{B}}^{\mathrm{L}}(\pi / 2+\delta \Lambda) \\
+M R_{\mathrm{A}}^{\mathrm{L}}(\pi / 2-\delta \Lambda)-M R_{\mathrm{B}}^{\mathrm{L}}(\pi / 2+\delta \Lambda),
\end{gathered}
$$

or simply

$$
\Delta I^{\mathrm{L}}\left(\Lambda_{\mathrm{k}}\right)=\Delta Z E^{\mathrm{L}}+\Delta M R^{\mathrm{L}} .
$$

For the trailing direction, by subtracting eq. (2) from eq. (4), we have

$$
\Delta I^{\mathrm{T}}\left(\Lambda_{\mathrm{k}}\right)=\Delta Z E^{\mathrm{T}}+\Delta M R^{\mathrm{T}} .
$$

Here, $\Delta$ means forming a differential of intensity over $\mathrm{B}$ to $\mathrm{A}$ for the leading direction and $\mathrm{A}$ to $\mathrm{B}$ for the trailing one. Since $\Delta Z E^{\mathrm{L}}$ and $\Delta Z E^{\mathrm{T}}$ are due to the same material, it may not be necessary to specify 'leading' or 'trailing'. The extra differential brightness can be specified in terms of number density $n\left(R_{\circ}\right)$, mean absorption cross-section $\sigma_{\mathrm{abs}}(\lambda)$ at wavelength $\lambda$, and temperature $T\left(R_{\mathrm{o}}\right)$ of the IPDs at heliocentric distance $R_{\mathrm{o}}$, to be precise, at $R_{\mathrm{O}} \cos \delta \Lambda$ from the Sun:

$$
\Delta Z E=n\left(R_{\mathrm{o}}\right) \sigma_{\mathrm{abs}}(\lambda) B_{\lambda}\left(T\left[R_{\mathrm{o}}\right]\right) 2 R_{\mathrm{o}} \delta \Lambda,
$$

where $B_{\lambda}(T)$ means the Planck function for black body intensity. For the case of circular orbit $R_{\mathrm{O}}=1 \mathrm{AU}$. If the differentials of the MMR feature are negligibly small, eq. (8) will enable us to retrieve the local IPD emissivity, $n\left(R_{\mathrm{o}}\right) \sigma_{\mathrm{abs}}(\lambda) B_{\lambda}\left(T\left[R_{\mathrm{o}}\right]\right)$, directly from the observed differences, $\Delta I^{\mathrm{L}}$ and $\Delta I^{\mathrm{T}}$. It was Dumont (1973) who first pointed out the usefulness of differential brightness of ZL over finite elongation interval.

\section{(b) Differential Brightness due to the MMR Feature}

The DIRBE/COBE observations suggest that the clump of dusts partially locked within the Earth's mean motion resonance in the leading direction is centered at solar elongation $80^{\circ}$ (Reach et al. 1995), which is consistent with the picture emerging from dynamical simulations by Dermott et al.(1994). The newly reduced ZL map (Kwon et al. 2004) doesn't show any significant enhancement in brightness at $\epsilon \simeq 90^{\circ}$ in the leading direction. The two sight lines involved in the $\Lambda$-differentiation seems to be well off the leading clump. We therefore expect the $\Delta M R^{\mathrm{L}}$ to be negligibly small compared to $\Delta I^{\mathrm{L}}$. Even if outskirts of the clump touch the two sight lines, the clump is likely to render to the two sight lines the same column density, since they are apart at maximum $2 \delta \Lambda$ in elongation.

On the trailing side the MMR clump seems to be centered right at elongation $90^{\circ}$ (Reach et al. 1995), encompassing the two sight lines. However, its differential contribution $\Delta M R^{\mathrm{T}}$ to $\Delta I^{\mathrm{T}}$ is expected to be 
small again. According to the DIRBE/COBE observations, the FWHM of the trailing clump is as wide as $15^{\circ}$ and our viewing directions are positioned moreor-less symmetrically with respect to the central line, making $M R_{\mathrm{A}}^{\mathrm{T}}(\pi / 2+\delta \Lambda)$ and $M R_{\mathrm{B}}^{\mathrm{T}}(\pi / 2-\delta \Lambda)$ almost the same. Consequently $\Delta M R^{\mathrm{T}}$ is expected to be negligible to the directly observed differential brightness $\Delta I^{\mathrm{T}}$ itself. Question then is how small it might be. In other words we wonder whether there is any means to isolate $\Delta Z E^{\mathrm{T}}$ in the observed brightness difference, $\Delta I^{\mathrm{T}}$.

A first hand estimate comes from the difference, $\Delta I^{\mathrm{T}}-\Delta I^{\mathrm{L}}$, which is equal to $\Delta M R^{\mathrm{T}}-\Delta M R^{\mathrm{L}}$. Since $\Delta M R^{\mathrm{L}}$ is expected to be much smaller than $\Delta M R^{\mathrm{T}}$, from the difference between the two directly observed quantities, we will have a good estimate of the MMR contribution to the differential ZE brightness. If the dusts locked in the MMR are of optically different species from the general IPDs, the degree of MMR contamination may be judged by comparing colors of $\Delta I^{\mathrm{L}}$ and $\Delta I^{\mathrm{T}}$; the former is almost free from the MMR contribution and the latter could have been affected by the MMR clumps. If a significant difference exists in color between the two directions, we should quantify the contamination seriously. Optical observations by wide-filed CCD cameras like WIZARD (Ishiguro et al. 2002) or WICZO (Kwon et al. 2004) may help us to make quantitative estimate on its relative importance or unimportance, if background and flattening corrections are made accurately to night sky images taken by the camera. From the resulting CCD images of the $Z \mathrm{~L}$, we calculate the ratio of $Z L_{\mathrm{MMR}}^{\mathrm{T}}(\pi / 2-\delta \Lambda)-Z L_{\mathrm{MMR}}^{\mathrm{T}}(\pi / 2+\delta \Lambda)$ to $Z L_{\mathrm{MMR}}^{\mathrm{T}}(\pi / 2)$. After additional corrections are made, on the ZL brightness at different elongations, of the effect of the scattering phase function, the resulting ratio of the $\mathrm{ZL}$ brightness represents the ratio of column densities over the three light paths in the MMR clump. Therefore, this ratio can be also applied to the IR brightness of the MMR features. To make ultimate corrections we are to wait until the circumsolar dust ring locked in the Earth's MMR may have been probed by the Spitzer Space Telescope mission (Werner et al. 2004; Jayaraman et al. 2003).

In summary we will ignore the differential brightness due to the MMR clumps from eqs. (6) and (7), and assume that the brightness difference resulted from the $\Lambda$-differentiation is solely due to the local IPDs on chord $\overline{A B}$. It may not be necessary to include the MMR term at least in this stage of outlining strategies of the $\Lambda$-differentiation. In the last part of the paper a brief discussion will be given on how we may include the MMR dusts into the local emissivity measurement. Whether correction for the MMR feature is necessary will be decided after having the data from ASTRO-F and wide-field cameras.

\section{(c) Circular Orbit, Elliptical Motion, and Max- imum Density Plane}

Since the MMR contributions are ignored in both directions, it is not necessary to distinguish differential brightness $\Delta I^{\mathrm{L}}\left(\Lambda_{\mathrm{k}}\right)$ in the leading direction from that $\Delta I^{\mathrm{T}}\left(\Lambda_{\mathrm{k}}\right)$ in the trailing one. If ASTRO-F orbits the hypothetical Earth, the differential brightness remains the same over a whole year period. In that case, what we will have is only the mean volume emissivity, $n_{\mathrm{o}} \sigma_{\mathrm{abs}}(\lambda) B_{\lambda}\left(T_{\mathrm{o}}\right)$, of the IPDs at fixed heliocentric distance $R_{\mathrm{O}}$ (cf. eq. [8]). Although degeneracy of the density, cross-section and temperature hidden in the local emissivity is difficult to disentangle, this will comprise the first direct measurement of the emissivity. With direct we want to emphasize that the resulting emissivity is free from three dimensional models of the IPD cloud. All the previous estimates of the local emissivity depend on the chosen power-law exponents for density and temperature, and also on the local temperature $T_{0}$. Therefore, the local emissivity resulting from the $\Lambda$-differentiation will serve a strong observational criterion for IPD cloud models. If $T_{\mathrm{o}}$ is known, the emissivity measured at different wavelengths will further serve a composition indicator for IPDs.

For a number of reasons, in reality, the observed brightness difference will vary with $\Lambda_{\mathrm{k}}$ in a systematic fashion. In fact witnessing and deciphering all the possible modulations in $\Delta I\left(\Lambda_{\mathrm{k}}\right)$ is the whole purpose of this study. We may think of at least three modulation sources: (a) non-zero ellipticity of the Earth's orbit, (b) tilt of the maximum density plane of the IPD cloud with respect to the ecliptic plane, and (c) possible offcentering of the IPD cloud from the Sun.

The eccentricity and tilt modulations can be quantified as follows. Let us consider the eccentricity part first. Introducing a power-law relation of exponent $-\nu$ and $-\delta$ for the heliocentric variations of the dust number density and temperature, respectively, we may have from eqs. (6), (7) and (8)

$$
\begin{aligned}
& {\left[\frac{\Delta I_{\lambda}\left(\Lambda_{\mathrm{k}}\right)}{2 R \delta \Lambda}\right] \simeq \sigma_{\mathrm{abs}}(\lambda) n_{\mathrm{\circ}}} \\
& \quad \times\left[1-e_{\oplus} \cos \left(\Lambda_{\mathrm{k}}-\varpi_{\oplus}\right)\right]^{-\nu} \\
& \quad \times B_{\lambda}\left(T_{\mathrm{o}}\left[1-e_{\oplus} \cos \left(\Lambda_{\mathrm{k}}-\varpi_{\oplus}\right)\right]^{-\delta}\right),
\end{aligned}
$$

where $2 R \delta \Lambda$ is the chord length covering the observing session $\Lambda_{\mathrm{k}}, n_{\mathrm{o}}$ means IPD number density at heliocentric distance $R_{\mathrm{o}}$, which is equal to the semimajor axis of the Earth's elliptical orbit with eccentricity $e_{\oplus}$, and $\varpi_{\oplus}$ represents longitude of the Earth's perihelion. The observed brightness difference divided by the chord length is nothing but the mean volume emissivity, $\mathcal{E}_{\lambda}(R)$, of the IPDs at heliocentric distance $R$. Because $R$ can be calculated from $\Lambda_{\mathrm{k}}$, the left hand side of the equation is a directly observable quantity. We approximate the Planck function simply as $B_{\lambda}\left(T_{0}\right)\left[1-e_{\oplus} \cos \left(\Lambda-\varpi_{\oplus}\right)\right]^{-\alpha \delta}$, or further as $B_{\lambda}\left(T_{\mathrm{o}}\right)\left[1+\alpha \delta e_{\oplus} \cos \left(\Lambda-\varpi_{\oplus}\right)\right]$, where parameter $\alpha$ is 
a function of temperature $T_{\mathrm{o}}$ for given wavelength and can be calculated from

$$
\alpha\left(T_{\mathrm{o}} ; \lambda\right)=\left[\frac{d \ln B_{\lambda}(T)}{d \ln T}\right]_{T_{\mathrm{o}}} .
$$

Eq. (9) now becomes

$$
\mathcal{E}_{\lambda}(\Lambda) \simeq \mathcal{E}_{\lambda}^{\mathrm{o}}\left[1+(\alpha \delta+\nu) e_{\oplus} \cos \left(\Lambda-\varpi_{\oplus}\right)\right],
$$

where $\mathcal{E}_{\lambda}^{\mathrm{o}}=n_{\mathrm{o}} \sigma_{\text {abs }}(\lambda) B_{\lambda}\left(T_{\mathrm{o}}\right)$ is the mean volume emissivity for wavelength $\lambda$ of the IPDs at reference distance $R_{\mathrm{o}}$ from the Sun.

It is not possible to know $\alpha$, because $T_{\mathrm{o}}$ is not known a priori. Although observational data will decide the sum, $\alpha \delta+\nu$, the product of $\alpha$ and $\delta$ will take different values depending on the wavelength. If the wavelength under consideration is close to the one, at which the Planck function becomes maximum, we expect the product to be about two. This is because the amount of heating energy decreases with heliocentric distance as $R^{-2}$, the total amount of cooling will decrease with $R$ in the same fashion as the heating. If the wavelength is much longer than the maximum Planck wavelength, the parameter becomes unity and the product $\alpha \times \delta$ takes value much less than two, since the cooling done at such long wavelength is going to be very small in comparison to the one around the maximum of Planck function. If the wavelength is much shorter than the maximum yielding wavelength, the product can be quite large. But eccentricity of the Earth's orbit keeps amplitude $(\alpha \delta+\nu) e_{\oplus}$ still much less than unity, making eq. (11) an accurate description of the modulation.

The tilt of the maximum density plane of the IPD cloud with respect to the ecliptic plane will introduce another cosine wave modulation, again with a small amplitude but with a half year period, and with ecliptic longitude of the plane's ascending node being the phase angle. By imposing the tilt modulation of the form, $\left\{1+e_{\text {sym } \lambda} \cos \left(2\left[\Lambda-\Omega_{\text {sym }}\right]\right)\right\}$, upon eq. (11), we have

$$
\begin{aligned}
& \mathcal{E}_{\lambda}(\Lambda) \simeq \mathcal{E}_{\lambda}^{\circ} \cdot\left\{1+(\alpha \delta+\nu) e_{\oplus} \cos \left(\Lambda-\varpi_{\oplus}\right)\right. \\
& \left.\quad+e_{\mathrm{sym} \lambda} \cos \left(2\left[\Lambda-\Omega_{\mathrm{sym}}\right]\right)\right\} .
\end{aligned}
$$

Amplitude $e_{\text {sym } \lambda}$ of the tilt modulation for given wavelength should depend on the vertical density stratification of the IPD cloud. It is difficult to pin-down its value a priori, but not necessary to do so at this stage. Observational data will determine the value for us later. All we need at the moment is to check whether the amplitude is small. The tilt modulation amplitude depends on inclination $i$ of the maximum density plane and is in the order of $\sin i$, which amounts to a couple of percents at most. It is indeed small.

By taking $\Lambda$-differentiation observationally at a number of $\Lambda_{\mathrm{k}}$-positions, we first measure $\mathcal{E}_{\lambda}\left(\Lambda_{\mathrm{k}}\right)$ as a function of the Earth's ecliptic longitude, i.e. of the heliocentric distance. We then fit the resulting set of emissivity measurements in two selected wavelengths to the cosine-modulations, and determine the modulation parameters for each wavelength. In actual process of the least squares analysis, instead of using eq. (12), we employ

$$
\begin{aligned}
\mathcal{E}_{\lambda}(\Lambda) & \simeq \mathcal{E}_{\lambda}^{\circ}\left(1-e_{\oplus}^{2}\right)^{-(\alpha \delta+\nu)} \\
& \times\left[1+e_{\oplus} \cos \left(\Lambda-\varpi_{\oplus}\right)\right]^{(\alpha \delta+\nu)} \\
& \times\left\{1+e_{\mathrm{sym} \lambda} \cos \left(2\left[\Lambda-\Omega_{\mathrm{sym}}\right]\right)\right\}
\end{aligned}
$$

and deduce the following pieces of information: $(a)$ the mean volume emissivity, $n_{\mathrm{o}} \sigma_{\mathrm{abs}}(\lambda) B_{\lambda}\left(T_{\mathrm{o}}\right)$, of the IPDs at reference distance 1AU from the Sun, $(b)$ the sum, $(\alpha \delta+\nu)$, of Planck function weighted power-law indices, (c) the longitude, $\Omega_{\mathrm{sym}}$, of ascending node of the maximum density plane of the IPD cloud, and finally $(d)$ the amplitude, $e_{\operatorname{sym} \lambda}$, of the plane's tilt modulation. The items $(c)$ and $(d)$ will help us locate the IPD cloud central plane with respect to the ecliptic. The principal objectives of this study are at $(a)$ and $(b)$, from which we want to fix $\nu, \delta$, and $T_{\mathrm{o}}$ without introducing three dimensional density model of the IPD cloud.

\section{(d) Decoupling of the $\left(\nu, \delta, T_{\mathrm{o}}\right)$ Degeneracy}

From the monitoring of $\mathcal{E}_{\lambda}(\Lambda)$ over a year or so period, at two wavelengths, say $\lambda_{m}$ and $\lambda_{n}$, we first determine the reference emissivities for the wavelengths, which may relate the mean temperature, $T_{\mathrm{o}}$, of the IPDs at the reference position to the ratio of the absorption cross-sections as follows:

$$
\left(\frac{\mathcal{E}_{\lambda_{m}}^{\mathrm{o}}}{\mathcal{E}_{\lambda_{n}}^{\mathrm{o}}}\right)=\left[\frac{\sigma_{\mathrm{abs}}\left(\lambda_{m}\right)}{\sigma_{\mathrm{abs}}\left(\lambda_{n}\right)}\right] \frac{B_{\lambda_{m}}\left(T_{\mathrm{o}}\right)}{B_{\lambda_{n}}\left(T_{\mathrm{o}}\right)} .
$$

If the ratio in square brackets on the right hand side of this equation is known, the reference temperature can be immediately calculated from it. However, the ratio is unknown to us, unless dust model is introduced.

Unlike the reference emissivity, the measured local emissivity may not be directly proportional to the Planck function with local dust temperature, because the observed emissivity modulation ought to have some contribution from the maximum density plane's tilt. Depending on how far the Earth is from the maximum plane at the time of observation, the local IPD number density changes. Therefore, before relating the observed emissivities to the Planck function, the tilt modulation should be corrected for. This will be done by the second term in parentheses on the left hand side of the following equation:

$$
\begin{gathered}
\frac{\mathcal{E}_{\lambda_{m}}(\Lambda)}{\mathcal{E}_{\lambda_{n}}(\Lambda)}\left(\frac{1+e_{\mathrm{sym} \lambda_{n}} \cos \left(2\left[\Lambda-\Omega_{\mathrm{sym}}\right]\right)}{1+e_{\mathrm{sym} \lambda_{m}} \cos \left(2\left[\Lambda-\Omega_{\mathrm{sym}}\right]\right)}\right) \\
=\left[\frac{\sigma_{\mathrm{abs}}\left(\lambda_{m}\right)}{\sigma_{\mathrm{abs}}\left(\lambda_{n}\right)}\right] \frac{B_{\lambda_{m}}(T)}{B_{\lambda_{n}}(T)} .
\end{gathered}
$$

In actuality $e_{\mathrm{sym} \lambda_{m}}$ will be equal to $e_{\mathrm{sym} \lambda_{n}}$, and hence we may not need to carry out the correction explicitly 
though. Since the ratio of cross-sections is not known, we substitute eq. (14) for the ratio and finally have

$$
\left(\frac{\mathcal{E}_{\lambda_{m}}(\Lambda) / \mathcal{E}_{\lambda_{m}}^{\mathrm{o}}}{\mathcal{E}_{\lambda_{n}}(\Lambda) / \mathcal{E}_{\lambda_{n}}^{\mathrm{o}}}\right)=\left[\frac{B_{\lambda_{n}}\left(T_{\mathrm{o}}\right)}{B_{\lambda_{m}}\left(T_{\mathrm{o}}\right)}\right] \frac{B_{\lambda_{m}}(T)}{B_{\lambda_{n}}(T)} .
$$

The year-long monitoring will determine the left hand side of this equation as a function of $\Lambda$, or of heliocentric distance. Therefore, the power-law exponent can be fixed of the heliocentric distance dependence of the mean dust temperature, if $T_{0}$ is given.

The simplest way of de-coupling the temperaturedensity degeneracy (cf: Reach 1991) in $(\alpha \delta+\nu)$ is to apply non-linear least squares fit of the above equation to the data, which will optimize reference temperature $T_{\mathrm{o}}$ and power-law index $\delta$. With the resulting $T_{\mathrm{o}}$ eq. (10) will fix $\alpha\left(T_{\circ} ; \lambda\right)$ for each of the two wavelengths, which will then give us two possible values of the powerlaw exponent, $\nu$, for the density distribution. If the two values agree with each other within a preset criterion, the degeneracy has been de-coupled, and we will know $\nu, \delta$ and $T_{\mathrm{o}}$ separately.

The non-linear least squares analysis may fail to give us $T_{\mathrm{o}}$ and $\delta$ together. Even if it gives, the resulting two sets for the two. wavelengths may not yield the same $\nu$ value. In these cases, we take the reference temperature as a free parameter, and obtain the index $\delta$ for given $T_{o}$ by fitting the data to eq. (16). Repeating the analysis with different choices of $T_{\mathrm{o}}$, we determine the temperature exponent $\delta\left(T_{0}\right)$ as a function of the reference temperature. Since $\alpha\left(T_{0} ; \lambda\right)$ can also be calculated from eq. (10), the power-law exponent for density distribution is automatically determined as a function of the reference temperature, namely from $\nu\left(T_{\mathrm{o}} ; \lambda\right)=(\nu+\alpha \delta)-\alpha\left(T_{\mathrm{o}} ; \lambda\right) \delta\left(T_{\mathrm{o}}\right)$. Please note that two values are available for the sum $(\nu+\alpha \delta)$ : One is from the least squares fit to $\mathcal{E}_{\lambda_{m}}(\Lambda)$ and the other from the fit to $\mathcal{E}_{\lambda_{n}}(\Lambda)$ (eq. [13]). Therefore, we expect to have two $\nu$ vs $T_{\mathrm{o}}$ curves. If they meet at certain $T_{\mathrm{o}}$, the reference temperature would be fixed, and hence the exponents $\nu$ and $\delta$. On the other hand, if the two curves have almost the same slope or are independent of parameter $T_{\mathrm{o}}$, it may not be possible to de-couple the degeneracy.

Which of these is the actual case for us is going to be determined mostly by the data quality and to some degree by the selection of color base. The data quality is likely to be out of our control; but some choices can be made for the color base. If both wavelengths of the given color base are in the same wavelength range where the Wien's approximation holds, we may have $\alpha\left(T_{\mathrm{o}} ; \lambda\right) \simeq(\mathrm{hc} / \lambda \mathrm{k})\left(1 / T_{\mathrm{o}}\right)$ for both of them (eq. [10]). This will make it hard to break the $\delta\left(T_{\mathrm{o}}\right) / T_{\mathrm{o}^{-}}$ degeneracy (Kelsall et al. 1998). If they are in the range of the Rayleigh-Taylor approximation, $\alpha\left(T_{0} ; \lambda\right)$ becomes unity. Consequently, the density index, $\nu$, becomes simply proportional to $\delta\left(T_{\mathrm{o}}\right)$ for the both wavelengths. This will lead the two lines run parallel and keep the degeneracy tight. By selecting wavelength

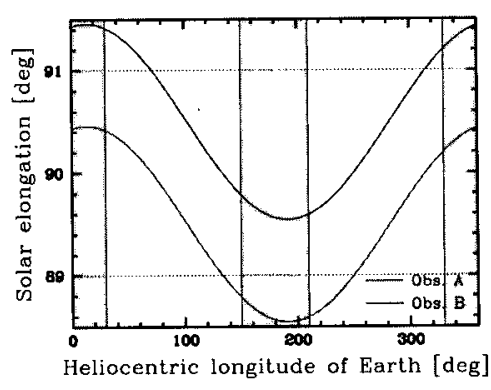

Fig. 2.- Constraints on the selection of observing sessions. Because Earth moves in an elliptical orbit, .the solar elongation angles of the leading direction at position $\mathrm{A}$ (shown in red) and B (in blue) differ from session to session. The same can be said for the trailing direction. For the hypothetical circular orbit, the leading direction has the same elongation angle, say at $89.5^{\circ}$, and the trailing direction does at $90.5^{\circ}$ all through the observing sessions. The grey areas demarcate the range of ecliptic longitude where the Milky Way crosses the ecliptic plane.

from each of the two approximation ranges, the two different behaviors of $\alpha$ can be utilized in breaking up the degeneracy.

\section{REQUIRED SENSITIVITY AND SELEC- TION OF OBSERVING SESSIONS}

\section{(a) Sensitivity Requirement for the $\Lambda$-Differentiation}

Before embarking on model simulations for feasibility test, we should check whether the IRC detection sensitivity is adequate for making the $\Lambda$-differentiation. If one sigma level of noise is less than about 1/50 of the maximum modulation amplitude in $\triangle Z E$, we may safely de-modulate the annual monitoring data of the local emissivity. Although arbitrary this criterion may be, it corresponds to $0.0014 \mathrm{MJy} \mathrm{sr}^{-1}$ for $12 \mu \mathrm{m}$ and $0.0012 \mathrm{MJy} \mathrm{sr}^{-1}$ for $25 \mu \mathrm{m}$. According to the latest, by the time of this writing, update of the IRC sensitivity given in the ASTRO-F Observer's manual, after $4 \times 4$ binning, the one sigma level of the IRC S11 band detection limit becomes $0.0378 \mathrm{MJy} \mathrm{sr}^{-1}$ and that of L24 does $0.0988 \mathrm{MJy} \mathrm{sr}^{-1}$. If we follow recommendations of the observer's manual, $64 \times 64$ binning is expected to reduce the noise to $1 / 64$ of the $4 \times 4$ binning values, which amounts to $0.0006 \mathrm{MJy} \mathrm{sr}^{-1}$ for S11 and $0.0015 \mathrm{MJy} \mathrm{sr}^{-1}$ for L24. And the $64 \times 64$ binning renders us angular resolution of $2.5 \mathrm{arcm} . \times 2.5 \mathrm{arcm}$., which is quite adequate for the studies of $\mathrm{ZE}$. From these values we may conclude that by binning the IRC observations one can meet the sensitivity requirements. 


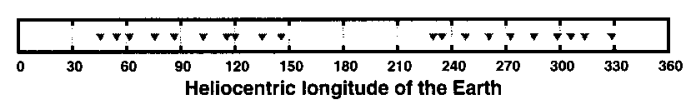

Fig. 3.- Twenty observing sessions are selected and marked with triangles at those ecliptic longitudes, where Earth would reside at the middle of each observing sessions. Since the selection should depends, among other factors, most strongly on the launching date, this is only meant to be an example.

\section{(b) Selection of Observing Sessions}

It is the Earth's elliptical orbit that enables us to determine the heliocentric distance dependence of the mean volume emissivity. However, eccentricity of the orbit introduces a complication in selecting the observing sessions for a year long monitoring. The complication is an acute one, because the range of lateral scan that ASTRO-F allows us is limited to at maximum $\pm 1.5^{\circ}$ from $90^{\circ}$ elongation. Because of this limitation there are additional forbidden zones in the ecliptic longitude coverage than the ones due to the Milky Way, Moon and South Atlantic Anomaly. In the case of the hypothetical circular orbit, the solar elongation angle of the leading direction at the first epoch observation (at A) remains the same, say at $89.5^{\circ}$, and the trailing direction at $90.5^{\circ}$, if $\delta \Lambda=0.5^{\circ}$ is taken. In the second epoch observation (at $\mathrm{B}$ ), they would remain at $90.5^{\circ}$ and $89.5^{\circ}$ for the leading and trailing directions, respectively. However, for the Earth's elliptical orbit, they depend on the observing session. We kept $\delta \Lambda$ to be $0.5^{\circ}$ and followed the solar elongation angles of the leading directions in the first and second epochs of each observing sessions. The results are shown, in Figure 2, for the first epoch in red and the second in blue.

We may not obtain high quality data on ZE in the region of the Milky Way, which is demarcated in the figure by grey area. Also removed are the regions where the solar elongation angle becomes larger than $91^{\circ}$ or less than $89^{\circ}$. This is a practical limit imposed by the spacecraft itself. If we reduce $\delta \Lambda$, the monitoring may cover wider range in the ecliptic longitude but with a sacrifice of observational accuracy. A differential step of $\delta \Lambda=0.5^{\circ}$ seems to be a reasonable compromise at the moment. With the visibility check utility for ASTROF, we have located Moon and the South Atlantic Anomaly, and selected 20 observing sessions along the ecliptic longitude. They are marked with red triangles in Figure 3 . In the conspicuous gaps over $\Lambda$ from $150^{\circ}$ through $210^{\circ}$ and $\pm 30^{\circ}$ centered at $\Lambda=0^{\circ}$, the Milky Way passes the ecliptic plane.

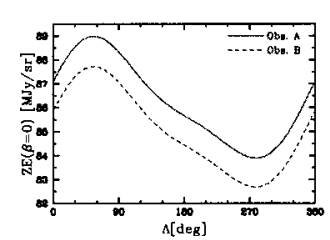

(a)

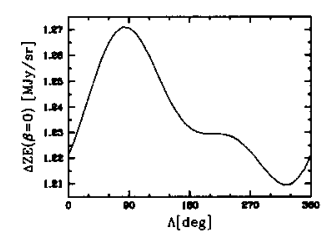

(c)

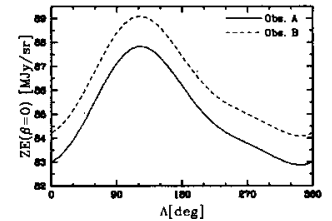

(b)

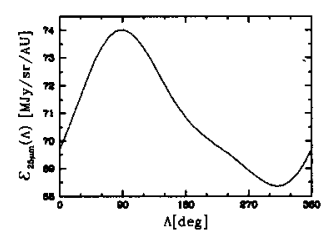

(d)
Fig. 4.- Steps of the $\Lambda$-differentiation are illustrated for $25 \mu \mathrm{m}$. In frame $(a)$ the orange solid line shows how the leading direction brightness measured at the first epoch varies with the time of observation, and the orange dotted line does the same for brightness measured at the second epoch. The same is done in frame $(b)$ for the trailing direction. The differential brightness is constructed by taking difference between the solid and dotted lines for the leading and trailing directions each. As shown in frame $(c)$ the differentials obtained from the leading and trailing directions turn out exactly the same. The differential brightness is converted, in frame $(d)$, to the mean volume emissivity.

\section{FEASIBILITY TEST FOR THE $\Lambda$-DIFFERENTIATION}

\section{(a) Synthesis of the Zodiacal Emission}

To prepare a data base for feasibility test we have constructed an IPD cloud model with the cosine model of the 3D density distribution. For the location of the maximum density plane of the IPD cloud we assigned $i=2.3^{\circ}$ for its inclination angle and $\Omega_{\mathrm{sym}}=75^{\circ}$ for the longitude of its ascending node. For the mean volume absorption cross-section, $n_{\mathrm{o}} \sigma_{\mathrm{abs}}(\lambda)$, we took $4.0 \times 10^{-20} \mathrm{~cm}^{-1}, 9.7 \times 10^{-21} \mathrm{~cm}^{-1}, 1.3 \times 10^{-20} \mathrm{~cm}^{-1}$, and $8.5 \times 10^{-21} \mathrm{~cm}^{-1}$ for $7,12,25$, and $60 \mu \mathrm{m}$, respectively. Although the power-law exponent for IPD density distribution in the ecliptic plane is known to change slowly with heliocentric distance (cf. Kwon \& Hong 1998), we simply took $\nu=1$ in this feasibility test. The mean temperature of the IPDs, $T(r)$, is assumed to vary with heliocentric distance $r$ in a power-law fashion $T(r)=T_{\mathrm{o}}\left(r / r_{\mathrm{o}}\right)^{-1 / 2}$ with $T_{\mathrm{o}}=278 \mathrm{~K}$ and $r_{\mathrm{o}}=1 \mathrm{AU}$. We then examine whether the $\Lambda$-differentiation recovers all these pieces of input information from the synthesized brightness of $\mathrm{ZE}$ at a set of selected wavelengths.

The synthesized $25 \mu \mathrm{m}$ ZE brightness at ecliptic longitudes $\Lambda_{\mathrm{k}}-\delta \Lambda$ and $\Lambda_{\mathrm{k}}+\delta \Lambda$ in the leading direction vary with $\Lambda_{\mathrm{k}}$ as the curves in solid and dotted lines in Fig- 


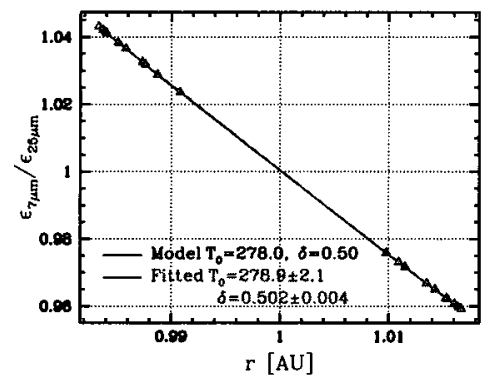

Fig. 5. - From the variation of the $7 \mu \mathrm{m}$-to- $25 \mu \mathrm{m}$ color with longitude one may determine the reference temperature and power-law exponent simultaneously. The colors obtained at the selected 20 observing sessions are shown in the ordinate, and the heliocentric distances of the spacecraft at the time of observation are in the abscissa. The $\Lambda$-differentiation retrieves the model parameters accurately.

ure $4 a$, respectively. The ordinates of the figure are in units of $\mathrm{MJy} \mathrm{sr}^{-1}$. The variation of $\mathrm{ZE}$ brightness with $\Lambda$ is a combined result of the eccentricity and tilt modulations. The first epoch measurement at A (solid line) should be brighter than the second at B (dotted line), because the former has an additional contribution from the IPDs that reside over $\overline{A B}$. Figure $4 b$ illustrates the same variation of ZE brightness in the direction trailing the Earth's motion. In the trailing direction the second epoch measurement is brighter than the first. The differential brightness between the two epochs is shown in Figure $4 c$ as a function of ecliptic longitude. Obviously it is exactly the same for the leading and trailing directions. We divide the differential $\mathrm{ZE}$ brightness by the chord length $2 R(\Lambda) \delta \Lambda$, and the resulting mean volume emissivity varies with longitude as in Figure $4 d$, whose ordinates are in units of $\mathrm{MJy} \mathrm{sr}^{-1} \mathrm{AU}^{-1}$. We have followed exactly the same steps of $\Lambda$-differentiation for the ZE brightness at $7 \mu \mathrm{m}, 12 \mu \mathrm{m}$, and also at $60 \mu \mathrm{m}$.

\section{(b) Without Imposing Noise}

¿From monitoring data of the emissivity modulation shown in Figures $4 d$ and of the similar ones for $7 \mu \mathrm{m}$ and $12 \mu \mathrm{m}$, we have constructed their ratios at the 20 selected $\Lambda_{\mathrm{k}}$ 's (Figure 3). An application of the least squares analysis to the resulting color determines the reference temperature $T_{\mathrm{o}}$ and the power-law index $\delta$ simultaneously. As compared in Figure 5, where the ordinates are the emissivity colors at the time of observation and the abscissae represent the heliocentric distance of the spacecraft at each observing sessions, the retrieved results agree satisfactorily with the model inputs. The deduced value for the longitude of the maximum density plane's ascending node turned out to be $74.8^{\circ}$, only slightly less than its input value. Its inclination cannot be determined from this type of analysis. We conclude that even the simplest non-linear least squares method would work satisfactorily, if the obser-

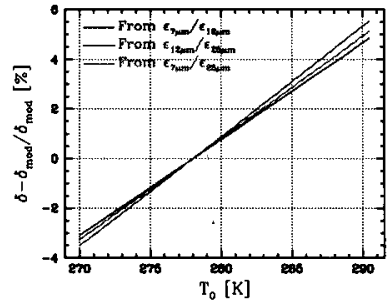

(a)

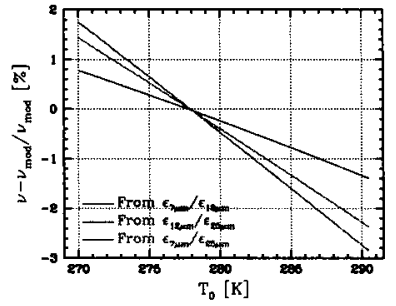

(b)
Fig. 6. - Adopting the reference temperature as a free parameter, we calculate the power-law indices of the temperature $(a)$ and density $(b)$ as functions of the parameter. The results from three pairs of color base all pass through one point, namely at $T_{\circ}=278 \mathrm{~K}$ and $\delta=\delta_{\bmod }(=0.5)$, and $\nu=\nu_{\bmod }(=1.0)$.

vational errors are sufficiently small.

To have correct value for $\delta$ we actually calculated the distance from the Sun to the middle of chord $\overline{A B}$, not to the spacecraft nor to the Earth. Unless observation is done at perihelion or aphelion, the chord center may not be the position to which the heliocentric distance should be measured for $\delta$-derivation. Because the Earth's orbit is not a circle, at positions other than these two special points, particles lying on one side from the middle of the chord ought to be slightly hotter than the ones on the other half side. To assign a proper distance, in principle, one should take into account for the asymmetry in temperature distribution between the two half sides. We have checked how significant this effect could be to the determination of power-law exponent for temperature. With observational accuracies afforded by the IRC in the mid infrared bands, the asymmetry effects are totally negligible. There may be cases to worry about in near IR, where ZE is much sensitive to the IPD temperature. In reality the abscissae of Figure 5 represent the heliocentric distance of the chord center in units of AU.

It is harder to fix the reference temperature and the power-law exponent simultaneously than one by one in a sequence. It is particularly so, when the data are noisy. We thus take the reference temperature as a free parameter, and determine $\delta$ as a function of this parameter. With $T_{\mathrm{o}}$ being assigned, $\alpha$ can be calculated for given wavelength, which in turn fixes a value for $\nu$ from the sum $\alpha \delta+\nu$. The resulting $\delta$ 's and $\nu$ 's from three choices of different color bases are shown, respectively, in Figure $6 a$ and Figure $6 b$ as functions of parameter $T_{\mathrm{o}}$. In the figure the relative error in percents is given in the ordinate and the trial $T_{\mathrm{o}}$ in the abscissa. Please remember that model inputs for the exponents are $\delta_{\text {mod }}$ $=0.5$ and $\nu_{\text {mod }}=1.0$. All the trial relations for the temperature exponent $\delta$ meet each other at the point (278, 0 ), and so do the ones for the density exponent $\nu$. From the figure we may conclude that this method retrieves 


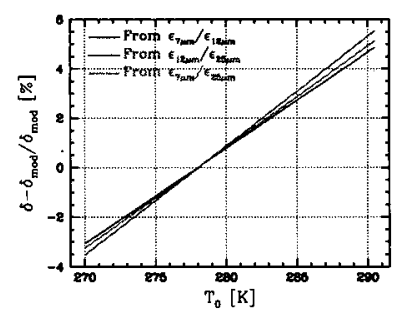

(a)

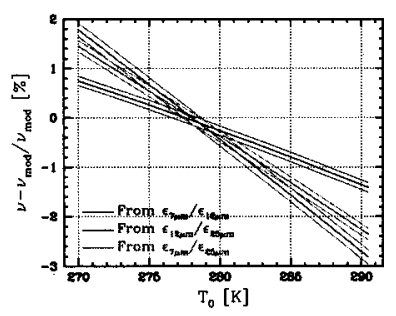

(b)
Fig. 7. - Same as in Figure 6 but noises corresponding to $128 \times 128$ binning are imposed upon the synthesized brightness of the zodiacal emission at each wavelength.

the input values of the two power-law exponents and the reference temperature accurately. Although observational noises are not included in this analysis, this test led us to have strong confidence on the retrieval strategy outlined in $\S 2$.

\section{(c) With Noise Imposed}

Upon the synthesized ZE brightness we have imposed random errors, whose magnitude corresponds to one sigma noise of the IRC with $128 \times 128$ binning. In Table 1 are listed the ZE brightness at the three wavelengths and corresponding one sigma noise for the cases of $32 \times 32,64 \times 64$ and $128 \times 128$ binnings. The reference temperature was taken again as an analysis parameter and the temperature exponent was determined as a function of the parameter. Results are shown in Figure $7 a$, where the ordinates represent the relative error in percents and the abscissae do the trial value for the reference temperature. From the figure we can determine the temperature and exponent quite accurately, since the three points met by the three curves occupy a narrow region in the $\left(T_{0}, \delta\right)$ plane. Not to make the figure too cluttered, we haven't shown error range for each line of the three color bases.

By assigning trial values to $T_{\mathrm{o}}$ we calculate $\alpha\left(T_{\mathrm{o}}\right)$ and with the corresponding $\delta\left(T_{\mathrm{o}}\right)$ already obtained we may then fix $\nu\left(T_{0}\right)$. The results are shown in Figure $7 b$, where the range of errors are given by a pair of thin lines for each of the three color bases. Since the points met by three thick lines reside in a reasonably small area in the plane, one may fix the power-law exponent of the density distribution from the figure with confidence. In Table 2 we have illustrated step by step how the unknown power-law indices and the reference temperature are determined from monitoring of the local mean volume emissivity at two chosen wavelengths. On the left side of the table are listed the results obtained from the data without noise, and the right part from the noiseimposed data. The retrieved information agrees very well with all the model inputs, including the ascending node of the maximum IPD density plane.

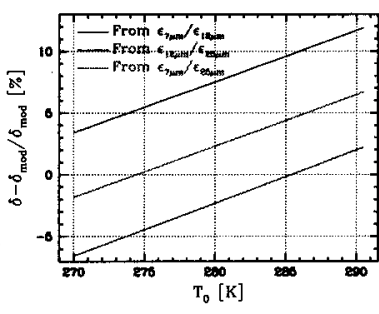

(a)

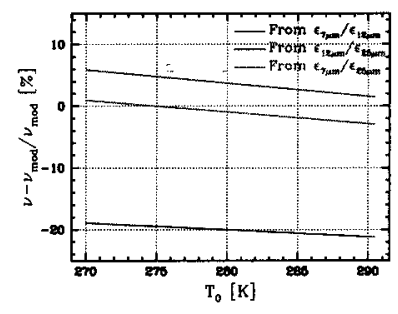

(b)
Fig. 8.- Same as in Figure 7 but imposed noises are from the $32 \times 32$ binning.

In order to demonstrate how important it is to keep the noise level low, we have constructed the same set of data but with $32 \times 32$ binnings. As shown in Figures $8 a$ and $8 b$ for $\delta$ and $\nu$, respectively, the same analysis completely failed to recover the input parameters. This failure was expected from the comparison of the noise level listed in Table 1 and the 1/50 criterion given in $\S 3$. We made a similar test with the $64 \times 64$ binning. As was expected from the same comparison, the results turn out to be very satisfactory.

\section{DISCUSSION AND CONCLUSION}

We have tried a number of combinations for color base, and noticed that the best determination can be made by the $7 \mu \mathrm{m}$ and $25 \mu \mathrm{m}$ pair. We may think of two reasons for the success of this wavelength pair. Firs of all, one of the two wavelengths is in the region of Wien's approximation, and the other in the Rayleigh-Jeans regime. Furthermore, in these bands one can make best measurements of $\mathrm{ZE}$ brightness with the IRC. It is important to keep the relative error in the same order of magnitude for different wavelengths. Whenever $60 \mu \mathrm{m}$ was included in forming the color base, we were unsuccessful to fix the unknown emissivity parameters. This is because the sensitivity of FIS aboard the ASTRO-F has some limitations for accurate measurement of the $\mathrm{ZE}$ at this long wavelength. Consequently the error involved in the $60 \mu \mathrm{m}$ band becomes unacceptably high in comparison to the IRC bands, and makes the non-linear least squares search a difficult task. In this study we haven't made the color corrections for the IRC bands, yet. This will be done shortly, when detailed information becomes available for the response function of each band.

Although we haven't included the MMR feature in our simulations, its existence won't harm the kind of local probing we are planning to do with the ASTRO$F$. The chord length extending 2 times the differential angle, $\delta \Lambda$, is about $0.017 \mathrm{AU}$ long and almost centered at Earth. On the other hand, even the trailing MMR blob, which is closer and denser than the leading one, seems to be about $0.1 \mathrm{AU}$ away from Earth (Dermott 
TABLE 1

Zodiacal Emission Brightness and Co-Added Errors

\begin{tabular}{c|c|ccc|c}
\hline \hline $\begin{array}{c}\text { Wavelength } \\
{[\mu \mathrm{m}]}\end{array}$ & $\mathrm{ZE}^{1}$ & \multicolumn{3}{|c|}{$1 \sigma$ Error [MJy/sr] } & ASTRO-F Sensitivity $^{2}$ \\
{$\left[\mathrm{MJy}_{\mathrm{sr}]}\right.$} & $32 \times 32$ & $64 \times 64^{-}$ & $128 \times 128$ & {$[\mathrm{MJy} / \mathrm{sr}]$} \\
\hline 7 & 26.6 & $1.34 \times 10^{-3}$ & $4.75 \times 10^{-4}$ & $1.68 \times 10^{-4}$ & $0.152(\mathrm{~S} 7$ band) \\
12 & 37.7 & $1.67 \times 10^{-3}$ & $5.91 \times 10^{-4}$ & $2.09 \times 10^{-4}$ & 0.189 (S11 band) \\
25 & 84.1 & $4.37 \times 10^{-3}$ & $1.41 \times 10^{-3}$ & $5.46 \times 10^{-4}$ & 0.494 (L24 band) \\
\hline \hline
\end{tabular}

${ }^{1}$ This corresponds to the ZE brightness at solar elongation $89^{\circ} .2$ in the ecliptic plane, when Earth is located at heliocentric ecliptic longitude $297^{\circ} .8$.

${ }^{2} 5 \sigma$-value for the $4 \times 4$ binning mode

TABLE 2

Determination of the Temperature and Density Exponents

\begin{tabular}{c|cc|cc}
\hline \hline \multirow{2}{*}{ Wavelength } & \multicolumn{2}{|c|}{ Without imposing noise } & \multicolumn{2}{c}{ With imposing noise } \\
& $7 \mu \mathrm{m}$ & $25 \mu \mathrm{m}$ & $7 \mu \mathrm{m}$ & $25 \mu \mathrm{m}$ \\
\hline$T_{\mathrm{o}}[\mathrm{K}]$ & \multicolumn{2}{|c|}{$278.9 \pm 2.1$} & \multicolumn{2}{c}{278.0 (fixed) } \\
$\delta$ & \multicolumn{2}{|c|}{$0.502 \pm 0.004$} & 2.346 & $0.504 \pm 0.0002$ \\
$\alpha$ & $7.375 \pm 0.056$ & $2.364 \pm 0.013$ & 7.375 \\
$\alpha \delta+\nu$ & $4.698 \pm 0.003$ & $2.184 \pm 0.003$ & $4.699 \pm 0.001$ & $2.185 \pm 0.001$ \\
$\nu$ & $0.991 \pm 0.042$ & $0.996 \pm 0.012$ & $0.996 \pm 0.002$ & $0.996 \pm 0.001$ \\
\hline$\Omega_{\text {sym }}\left[{ }^{\circ}\right]$ & $74.66 \pm 0.025$ & $74.90 \pm 0.025$ & $74.57 \pm 0.041$ & $74.92 \pm 0.080$ \\
$e_{\text {sym } \lambda}$ & $(9.5 \pm 0.01) \times 10^{-3}$ & $(9.6 \pm 0.01) \times 10^{-3}$ & $(9.5 \pm 0.02) \times 10^{-3}$ & $(9.6 \pm 0.03) \times 10^{-3}$ \\
\hline \hline
\end{tabular}

et al. 1994). Therefore, our differential chord may not touch the blob at all. If it does touch, we will simply include the MMR contribution to the local emissivity. This can be done as follows. The MMR features are located always in the same relative position in the frame rotating with the speed of Earth's mean motion, and the ASTRO-F will see the same particles while moving around the Sun with Earth. But heliocentric distance of these particles varies in the same manner as the heliocentric distance of the Earth, except small difference due to the Earth's epicyclic motion in the rotating frame. Therefore the local mean volume emissivity of the MMR portion will experience the same eccentricity modulation of the Earth. Consequently, an inclusion of the MMR contribution won't cause any conceptual difficulties nor practical ones to the analysis we are proposing here. It should be pointed out that the changing geocentric distance of the MMR particles due to the Earth's epicyclic motion will render only second order effects to the measurement of local emissivity.

The IRAC of the Spitzer Space Telescope has detection sensitivity more or less comparable to the IRC of the ASTRO-F, if enough number of pixel values can be co-added (http://ssc.spitzer.caltech.edu). Although we haven't checked the question of sensitivity quantitatively, the IRAC's smaller field of view than the IRC's may cause some difficulty in meeting the sensitivity requirement for the $\Lambda$-differentiation by bin adding. In principle one could make, with the Spitzer Space
Telescope, the same kind of $\Lambda$-differentiations we are proposing here. Since the Spitzer is allowed to point closer to the Sun than the ASTRO-F can, if enough sensitivity can be mustered by binning, it can probe much inner part of the IPD cloud than the Earth's immediate neighbors. Even if IRAC's sensitivity doesn't meet the sensitivity requirement, its wide coverage of the solar elongation angle would help one probe global $3 \mathrm{D}$ structure of the IPD cloud. It would be nice if the IRC/ASTRO-F and IRAC/Spitzer point simultaneously same regions of the interplanetary space at two different vantage points and coordinate their monitoring observations of the ZE modulation, particularly when the Spitzer is deep inside the trailing blob of the Earth's MMR dust ring (Jayaraman et al. 2003).

If we make full uses of the ASTRO-F's superb detection sensitivity, fine spatial resolution and firm calibration stability, with the $\Lambda$-differentiation method, we may retrieve the IR local emissivity, temperature of the local IPDs, and its dependence on heliocentric distance. At the same time we may also determine the power-law exponent for the heliocentric density distribution. The idea behind the $\Lambda$-differentiation is an old one in the field of zodiacal light studies, but the sensitivity of ground-based observations hasn't reached the level to make this type of analysis feasible. In the zodiacal emission, however, the ASTRO-F will make its implementation possible for the first time. 


\section{ACKNOWLEDGEMENTS}

This research was supported by a Korea Research Foundation grant (KRF-2001-015-DP0609). JP would like to acknowledge the support from KOSEF R142002-058-01003-0.

\section{REFERENCES}

Davies, J. K., Green, S. F., Stewart, B. C., Meadows, A. J., \& Aumann, H. H. 1984, The IRAS fast-moving object search, Nature, 309, 315

Dermott, S. F., Nicholson, P. A., Burns, J. A., \& Houck, J. R. 1984, Origin of the solar system dust bands discovered by IRAS, Nature, 312, 505

Dermott, S. F., Nicholson, P. D., Burns, J. A., \& Houck, J. R. 1985, An analysis of IRAS' solar system dust bands, in Properties and Interactions of Interplanetary Dust, ed. by R. H. Giese, P. Lamy, (D. Reidel:Dordrecht), p.395

Dermott, S. F., Jayaraman, S., Xu, Y. L., Gustafson, B. A. S., \& Liou, J. C. 1994, A circumsolar ring of asteroidal dust in resonant lock with the Earth, Nature, 369, 719

Dumont, R. 1965, Séparation des composantes atmosphériques et interplanétaire et stellaire du ciel nocturne à $5000 \AA$. Applications à la photométrie de la lumière zodiacale et du Gegenschein, Annales d'Astrophysique, 28, 265

Dumont, R. 1973, Phase Function and Polarization Curve of Interplanetary Scatterers from Zodiacal Light Photopolarimetry, Planetary \& Space Science, 21,2155

Dumont, R. 1975, On the gradient of zodiacal light with heliocentric distance, Planetary \& Space Science, 23, 1235

Dumont, R., Renard, J. B., Levasseur-Regourd, A. C. \& Weinberg, J. L. 1998, Disentangling the main populations of the zodiacal cloud from zodiacal light observations, EP\&S, 50, 473

Hong, S. S. 1985, Henyey-Greenstein Representation of the Mean Volume Scattering Phase Function for Zodiacal Dust, A\&Ap, 146, 67

Ishiguro, M., Nakamura, R., Fujii, Y., Morishige, K., Yano, H., Yasuda, H., Yokogawa, S., \& Mukai, T. 1999, First detection of visible zodiacal dust bands from ground-based observations, ApJ, 511, 432

Ishiguro, M., Watanabe, J., Usui, F., Tanigawa, T., Kinoshita, D., Suzuki, J., Nakamura, R., Ueno, M., \& Mukai, T. 2002a, First Detection of an Optical Dust Trail along the Orbit of 22P/Kopff, ApJ, 572, L117

Ishiguro, M., Mukai, T., Nakamura, R., Usui, F., \& Ueno, M. 2002b, WIZARD: New Observation system of zodiacal light in Kobe University, in Dust in the Solar System and Other Planetary Systems,
COSPAR COLLOQUIUM SERIES Volume 15, ed. by S.F. Green, I.P. Williams, J.A.M. McDonnell and N. McBride (Amsterdam:Pergamon), p. 98

Ishiguro, M., Kwon, S. M., Sarugaku, Y., Hasegawa, S., Usui, F., Nishiura, S., Hakada, Y., \& Yano, H. 2003 Discovery of the dust trail of the Stardust comet sample return mission target: $81 \mathrm{P} /$ Wild 2, ApJ, 589, L101

Jayaraman, S., Grogan, K., Reach, W., Stansberry, J., \& Werner, M. 2003, Proposed Observations of Earth's Resonant Ring and Zodiacal Cloud with SIRTF, American Astronomical Society, DPS meeting \#35, \#38.32

Kelsall, T., Weiland, J. L., Franz, B. A., Reach, W. T., Arendt, R. G., Dwek, E., Freudenreich, H. T., Hauser, M. G., Moseley, S. H., Odegard, N. P., Silverberg, R. F., \& Wright, E. L. 1998, The COBE diffuse infrared background experiment search for the cosmic infrared background. II. Model of the iInterplanetary dust cloud, ApJ, 508, 44

Kwon, S. M. \& Hong, S. S. 1998, Three-dimensional infrared models of the interplanetary dust distribution, EP\&S, 50, 501

Kwon, S. M., Hong, S. S., \& Weinberg, J. L. 2004, An observational model of the zodiacal light brightness distribution, NewA., 10, 91

Kwon, S. M., Hong, S. S. \& Shin, K. J. 2004, Development of Wide-field Imaging Camera for Zodiacal Light Observation(WICZO), submitted to JKAS

Levasseur-Regourd, A. C. \& Dumont, R. 1980, Absolute photometry of zodiacal light, A\&A, 84, 277

Low, F. J., Young, E., Beintema, D. A., Gautier, T. N., Beichman, C. A., Aumann, H. H., Gillett, F. C., Neugebauer, G., Boggess, N., \& Emerson, J. P. 1984, Infrared cirrus - New components of the extended infrared emission, ApJ, 278, L19-L22

Reach, W. T. 1991, Zodiacal emission. II - Dust near ecliptic, ApJ, 369, 529

Reach, W. T., Franz, B. A., Weiland, J. L., Hauser, M. G., Kelsall, T. N., Wright, E. L., Rawley, G., Stemwedel, S. W., \& Spiesman, W. J. 1995, Observational Confirmation of a Circumsolar Dust Ring by the COBE Satellite, Natur., 374, 521

Sykes, M. V. 1988, IRAS observations of extended zodiacal structures, ApJ, 334, L55

Sykes, M. V., \& Walker, R. G. 1992, Cometary dust trails. I - SurveyIcarus, 95, 180

Tanabe, H. 1963, Zodiacal Light and Airglow Components at $5300 \AA$, PASJ, 16, 324

Weinberg, J. L. 1963, Photoelectric Polarimetry of the Zodiacal Light at Lambda 5300, Ph. D. Thesis, University of Colorado

Werner, M., et al. 2003, The Spitzer Space Telescope Mission, ApJS, 154, 1 